\title{
Disziplinierung durch Leiharbeit? Neue Nutzungsstrategien von Leiharbeit und ihre arbeitspolitischen Folgen
}

Immer häufiger nutzen Betriebe das Flexibilisierungsinstrument Leiharbeit nicht mehr nur, um kurzfristig auf Auftragsspitzen oder Personalausfall zu reagieren, sondern um dauerhaft ein Sicherheitsnetz für die Kapitalrendite zu errichten. Mit dem Funktionswandel der Leiharbeit verändert sich auch die Stellung der Leiharbeitskräfte im Einsatzbetrieb: Dessen Belegschaft ist nicht mehr in eine Kernund eine Randbelegschaft gespalten; zumindest im Arbeitsprozess sind Stammkräfte und Leiharbeiter eng miteinander verflochten. Durch die neuen Nutzungsstrategien wird im Einsatzbetrieb ein Mechanismus etabliert, der vermittelt über die prekäre Position der Leiharbeitskräfte auch die langjährigen Stammkräfte stärker an die allgemeine Arbeitsmarktentwicklung koppelt.

\section{1 \\ Einleitung}

Mit dem Leiharbeitsboom der vergangenen Jahre ist auch das wissenschaftliche Interesse an der Beschäftigungsform Leiharbeit sprunghaft angestiegen. Die Nutzung des Flexibilisierungsinstruments Leiharbeit im Einsatzbetrieb wurde vor allem aus zwei Perspektiven untersucht: Einerseits sind Bestimmungsfaktoren der betrieblichen Nutzung von Leiharbeit herausgearbeitet worden, anderseits die arbeits- und mitbestimmungspolitischen Folgen des Einsatzes von Leiharbeit. Trotz einer $\mathrm{Zu}$ nahme der Nutzung im Dienstleistungssektor ist Leiharbeit noch immer vorwiegend ein Phänomen der Großbetriebe des verarbeitenden Gewerbes (Bellmann/Kühl 2007, S. 9ff.). Hinsichtlich der Einsatzformen und der Nutzungsmotive hat die Forschung eine erhebliche Heterogenität diagnostiziert (Promberger 2006, S. 265). Nichtsdestotrotz lassen sich zwei eng miteinander verbundene Entwicklungstrends identifizieren, die dem Leiharbeitsboom seit der Reform des Arbeitnehmerüberlassungsgesetzes (AÜG) 2004 zugrunde liegen - eine Zunahme der Intensivnutzung und ein Funktionswandel der Leiharbeit. Zum einen geht das Beschäftigungswachstum der Leiharbeit (vor der aktuellen Krise) zu einem erheblichen Teil auf das Wachstum des Segments der Intensivnutzer zurück. 2006 gehörte bereits jeder zehnte Einsatzbetrieb in die Kategorie der Intensivnutzer mit einem Leiharbeitsanteil an der Belegschaft von über $20 \%$ (Bellmann/Kühl 2007, S. 19ff.). Zum anderen lässt sich auch eine Verschiebung der Nutzungsmotive beobachten. Darauf weist die inzwischen verbreitete These vom „Funktionswandel der Leiharbeit" (Dörre/Holst 2007; Miegel et al. 2007; Seifert/Brehmer 2008) hin. Immer mehr Betriebe setzen Leiharbeitskräfte nicht mehr nur kurzfristig zur Abwicklung von Auftragsspitzen ein, sondern dauerhaft zur Absicherung der Kapitalrendite gegen das „Kapazitätsrisiko" der Absatzmärkte (Dudenhöffer/ Büttner 2006, S. 32).

Mitbestimmungspolitisch erweist sich als problematisch, dass Leiharbeiter seit 2001 zwar wahlberechtigt bei den Betriebsratswahlen sind, bei der Berechnung der Freistellungen jedoch nicht mitgezählt werden. Gerade in Betrieben mit einem hohen Leiharbeitsanteil wird die Handlungsfähigkeit der Betriebsräte dadurch strukturell beeinträchtigt. Zudem haben noch längst nicht alle Betriebsräte Leiharbeit als mitbestimmungspolitisches Thema für sich entdeckt und die Leiharbeitskräfte in ihre Interessenvertretungspraxis integriert (Wassermann/Rudolph 2007; Promberger 2006). Auch zu den arbeitspolitischen Folgen der Leiharbeitsnutzung liegen inzwischen mehrere Fallstudien vor. Leiharbeit ist als Beschäftigungsverhältnis mit einem hohen Prekaritätsrisiko ausgestattet, das disziplinierend und individualisierend auf die Leiharbeitskräfte wirkt (Vogel 2004; Dörre et al. 2004). Zudem wurde gezeigt, dass die Disziplinierungseffekte der Leiharbeit auch auf die formal sicheren Stammkräfte übergreifen können: Indem ihnen täglich ihre Ersetzbarkeit vor Augen geführt wird, wird zumindest in einigen Betrieben Druck auf das Stammpersonal ausgeübt (Kraemer/Speidel 2004; Brinkmann et al. 2006, S. 61f.; aktuell: Castel/Dörre 2009).

Auffällig ist jedoch, dass die Diskussion der arbeits- und mitbestimmungspolitischen Folgen eines Leiharbeitseinsatzes bislang nicht mit der Heterogenität der Einsatzformen und Nutzungsmotive in Verbindung gebracht worden ist. Dies ist umso überraschender, als es in der internationalen Forschung durchaus Hinweise darauf gibt, dass sowohl die Reichweite als auch die Beschaffenheit der möglichen Disziplinierungseffekte von der Art des betrieblichen Einsatzes der Leiharbeitskräfte abhängig sind (Broschak/Davis-Blake 2006; Beaud/Pialoux 2004, S. 55ff.). An diesem Punkt setzt der Beitrag an: Welche arbeitspolitischen Konsequenzen haben die neuen betrieblichen Nutzungsstrategien, die der These des Funktionswandels der Leiharbeit zugrunde liegen? Im Fokus der Analyse steht das Verhältnis zwischen Stammkräften und Leiharbeitern im Einsatzbetrieb. Gegliedert ist der Beitrag folgendermaßen: Nach einer kurzen Vorstellung des empirischen Materials (Abschnitt 2) wird eine Typologie betrieblicher Nutzungsstrategien von Leiharbeit präsentiert (Abschnitt 3). An zwei Betriebsfallstudien wer-

Hajo Holst, Wissenschaftlicher Mitarbeiter
an der Friedrich-Schiller-Universität Jena,
Institut für Soziologie, Lehrstuhl für
Arbeits-, Industrie- und Wirtschaftssoziologie.
Arbeitsschwerpunkte: International verglei-
chende industrielle Beziehungen, nicht-
standardisierte Beschäftigungsformen,
betriebliche Flexibilisierungsstrategien
e-mail: hajo.holst@uni-jena.de

Hajo Holst, Wissenschaftlicher Mitarbeiter an der Friedrich-Schiller-Universität Jen Arbeits-, Industrie- und Wirtschaftssoziologie. Arbeitsschwerpunkte: International vergleie-mail: hajo.holst@uni-jena.de 
den anschließend exemplarisch die arbeitspolitischen Auswirkungen von zwei der drei betrieblichen Nutzungsstrategien dargelegt (Abschnitte 4 und 5). Abschließend erfolgen eine zusammenfassende Diskussion der Ergebnisse und ein Ausblick auf zukünftigen Forschungsbedarf (Abschnitt 6).

\section{2 \\ Empirische Basis: Das Sample}

Empirisch basiert der Beitrag auf einem explorativ angelegten Forschungsprojekt, das sich gegenwärtig an der Friedrich-SchillerUniversität (FSU) Jena genauer mit den neuen Nutzungsstrategien der Leiharbeit und ihren arbeits- und mitbestimmungspolitischen Folgen auseinandersetzt. ${ }^{1}$ Den Kern des Samples bilden sechs Intensivbetriebsfallstudien aus der Metall- und Elektroindustrie (Großmaschinenbau, Elektromaschinenbau, Verbindungstechnik, Automobilzulieferer, Automobilhersteller, Energietechnik), für die wir insgesamt 72 Interviews mit Stammkräften (SK), Leiharbeitern (LA), direkten Vorgesetzen (DV), Betriebsräten $(\mathrm{BR})$ und Personalabteilungen (PA) geführt haben. Übersicht 1 stellt die Fallbetriebe mit den wichtigsten Kennzahlen (Betriebsgröße, Leiharbeitsanteil an der Belegschaft, Nutzungsstrategie, Interviews) kurz dar. Neben den Intensivfallstudien wurden sechs Kurzbetriebsfallstudien auf der Basis von Experteninterviews mit Betriebsräten erhoben, sodass insgesamt 78 Interviews geführt wurden. Drei der Betriebe zählen zur Metall- und Elektroindustrie (Automobilhersteller, Automobilzulieferer, Stahlwerk), die drei anderen stellen kontrastierende Fallstudien (Milchwirtschaftsbetrieb, Callcenter, Gebäckhersteller) aus anderen Branchen dar. Während von den Intensivfallstudien fünf Betriebe Leiharbeit strategisch nutzen und nur ein Betrieb dem Typus des Flexibilitätspuffers entspricht, ist das Verhältnis bei den Kurzfallstudien genau anders herum: Nur der Automobilhersteller nutzt Leiharbeit, um die Kapitalrendite gegen das Marktrisiko abzusichern, die anderen fünf Untersuchungsbetriebe nutzen Leiharbeit reaktiv, um auf reale Schwankungen des Auftragsvolumens zu reagieren.

\begin{tabular}{|c|c|c|c|c|}
\hline \multicolumn{5}{|c|}{ Übersicht 1: Untersuchungsbetriebe (Intensivbetriebsfallstudien) } \\
\hline Fallbetrieb & MA & $\begin{array}{c}\text { LA } \\
\text { in } \%\end{array}$ & Nutzungsstrategie & Interviews \\
\hline Automobilhersteller & 30.000 & 5 & strategische Nutzung & $1 \mathrm{BR}, 2 \mathrm{DV}, 4 \mathrm{LA}, 4 \mathrm{SK}$ \\
\hline Großmaschinenbau & 550 & 9 & Flexibilitätspuffer & $1 \mathrm{PA}, 2 \mathrm{BR}, 2 \mathrm{DV}, 4 \mathrm{LA}, 4 \mathrm{SK}$ \\
\hline Elektromaschinenbau & 650 & 18 & strategische Nutzung & $1 \mathrm{PA}, 1 \mathrm{BR}, 2 \mathrm{DV}, 4 \mathrm{LA}, 4 \mathrm{SK}$ \\
\hline Energietechnik & 180 & 15 & strategische Nutzung & $1 \mathrm{PA}, 1 \mathrm{BR}, 1 \mathrm{DV}, 4 \mathrm{LA}, 4 \mathrm{SK}$ \\
\hline Automobilzulieferer & 230 & 30 & strategische Nutzung & $1 \mathrm{PA}, 1 \mathrm{BR}, 2 \mathrm{DV}, 4 \mathrm{LA}, 4 \mathrm{SK}$ \\
\hline Verbindungstechnik & 950 & 21 & strategische Nutzung & $1 \mathrm{PA}, 1 \mathrm{BR}, 3 \mathrm{DV}, 4 \mathrm{LA}, 4 \mathrm{SK}$ \\
\hline \multirow{2}{*}{\multicolumn{5}{|c|}{$\begin{array}{l}M A=\text { Mitarbeiter; } L A=\text { Leiharbeiter; } B R=\text { Betriebsrat; } D V=\text { Direkte Vorgesetzte; } \\
P A=\text { Personalabteilung; } S K=\text { Stammkräfte. }\end{array}$}} \\
\hline & & & & VVI MITTEILUNGEN \\
\hline
\end{tabular}

\begin{tabular}{|c|c|c|c|}
\hline \multicolumn{4}{|c|}{$\begin{array}{l}\text { Übersicht 2: Betriebliche Nutzungsstrategien von Leiharbeit als } \\
\text { Flexibilisierungsinstrument }\end{array}$} \\
\hline & $\begin{array}{c}\text { Typ I } \\
\text { Ad-hoc-Ersatz }\end{array}$ & $\begin{array}{c}\text { Typ II } \\
\text { Flexibilitätspuffer }\end{array}$ & $\begin{array}{l}\text { Typ III } \\
\text { Strategische Nutzung }\end{array}$ \\
\hline \multicolumn{4}{|l|}{ Form } \\
\hline Nutzungsintensität & minimal & mittel bis hoch $(>5 \%)$ & mittel bis hoch $(>5 \%)$ \\
\hline Reichweite & punktuell & $\begin{array}{l}\text { begrenzt auf } \\
\text { Randbelegschaft }\end{array}$ & $\begin{array}{l}\text { umfassend } \\
\text { in allen Arbeits- } \\
\text { bereichen }\end{array}$ \\
\hline Dauer & temporär & temporär & verstetigt \\
\hline \multicolumn{4}{|l|}{ Funktion } \\
\hline Flexibilität & $\begin{array}{l}\text { Personalersatz } \\
\text { (Suchkosten) }\end{array}$ & $\begin{array}{l}\text { Schwankungen des } \\
\text { Auftragsvolumens } \\
\text { (Such- und } \\
\text { Einstellungskosten) }\end{array}$ & $\begin{array}{c}\text { Strategische } \\
\text { Ungewissheitskontrolle } \\
\text { (Entlassungskosten) }\end{array}$ \\
\hline Kostenreduktion & Lohnkosten & Lohnkosten & Lohnkosten \\
\hline $\begin{array}{l}\text { Verhältnis Stammkräfte - } \\
\text { Leiharbeitskräfte im } \\
\text { Arbeitsprozess }\end{array}$ & $\begin{array}{l}\text { punktuelle } \\
\text { Interaktionen }\end{array}$ & $\begin{array}{l}\text { Segmentierung in } \\
\text { Kern- und } \\
\text { Randbelegschaft }\end{array}$ & $\begin{array}{l}\text { Verflechtung von } \\
\text { Stamm- und } \\
\text { Leiharbeitskräften }\end{array}$ \\
\hline Quelle: Zusammenstellung des A & & \multicolumn{2}{|c|}{ WSI MITTEILUNGEN } \\
\hline
\end{tabular}

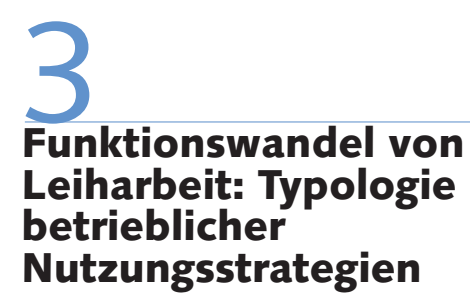

Die These vom Funktionswandel der Leiharbeit bezieht sich auf die Durchsetzung neuer betrieblicher Nutzungsstrategien. Seit der Reform des AÜG 2004 treten auch in Deutschland Nutzungsstrategien von Leiharbeit, die in der Vergangenheit nur aus den angelsächsischen Ländern, Frankreich und den Niederlanden bekannt waren, neben die bekannten Strategien (Beaud/Pialoux 2004; Carnoy et al. 1997; Theodore/Peck 2002). Von den etablierten Strategien unterscheiden sich die neuen Nutzungsstrategien sowohl hinsichtlich der Form des Einsatzes als auch hinsichtlich der Funktion der Leiharbeit. Übersicht 2 präsentiert eine Typologie betrieblicher Nutzungsstrategien. Diese haben wir in Auseinandersetzung mit der deutschen und der angelsächsischen Forschungsliteratur formuliert und in der empirischen
Forschung weiterentwickelt. Ausgehend von der Funktion der Leiharbeit als Flexibilisierungsinstrument lassen sich drei idealtypische Nutzungsstrategien identifizieren: der Ad-hoc-Ersatz, der Flexibilitätspuffer und die strategische Nutzung. Die drei Typen schließen sich keineswegs aus, sondern ergänzen sich. In der Praxis setzen Betriebe, die Leiharbeit strategisch nutzen, häufig auch Leiharbeitskräfte zur Abwicklung kurzfristiger Auftragsspitzen oder als Ersatz für unerwarteten Personalausfall ein. Entscheidend für die arbeitspolitischen Konsequenzen des Leiharbeitseinsatzes und damit auch für die Einordnung des Betriebs in die Typologie ist jedoch die im Betrieb dominierende Nutzungsstrategie.

Die Form des Leiharbeitseinsatzes bezieht sich auf die Nutzungsintensität, die Dauer und die Reichweite des Einsatzes. Die Nutzungsintensität erfasst den Anteil der Leiharbeitskräfte an der Gesamtbelegschaft des Einsatzbetriebs (Bellmann/Kühl

\footnotetext{
1 Gefördert wird das Projekt „Funktionswandel von Zeitarbeit " von der Otto-Brenner-Stiftung. Neben dem Autor ist Oliver Nachtwey an dem Projekt beteiligt, die Leitung hat Klaus Dörre.
} 
2007, S. 19). Die Dauer eines Leiharbeitseinsatzes schwankt zwischen zeitlich präzise eingegrenzten Kurzeinsätzen und einem faktisch unbefristeten, auf Dauer gestellten Einsatz. Dagegen beschreibt die Reichweite die Ausbreitung der Leiharbeitskräfte im Einsatzbetrieb: von einem punktuellen Einsatz, der auf wenige, sachlich und räumlich eng abgegrenzte Bereiche beschränkt bleibt, bis zu einem umfassendem Leiharbeitseinsatz, der sich auf (fast) alle Arbeitsbereiche erstreckt. Die Funktion des Leiharbeitseinsatzes beschreibt die Ziele, die von der Betriebsoder Unternehmensleitung mit dem Rückgriff auf das Flexibilisierungsinstrument Leiharbeit verfolgt werden. Die unterschiedlichen Funktionen bilden das Rückgrat der Typologie. Neben der numerischen Flexibilität bietet Leiharbeit den Einsatzbetrieben zudem die Möglichkeit, die Arbeitskosten zu reduzieren (Bellmann/ Kühl 2007, S. 16). Zum einen müssen nur die tatsächlich geleisteten Arbeitsstunden der Leiharbeitskräfte bezahlt werden, für die Lohnfortzahlung im Krankheitsfall und den gesetzlichen Urlaubsanspruch ist die Verleihfirma zuständig. Zum anderen können Einsatzbetriebe aufgrund der Regulierung der Leiharbeit ihre eigenen Tarifverträge umgehen.

\subsection{DER AD-HOC-ERSATZ}

Diese Nutzungsform stand am Anfang der Entwicklung der Leiharbeitsbranche (Theodore/Peck 2002, S. 467). Leiharbeit wird von den Einsatzbetrieben eingesetzt, um (kurzfristigen) Personalausfall zu kompensieren. Ziel des Leiharbeitseinsatzes ist es, trotz des unerwarteten Personalausfalls die Kontinuität der betrieblichen Abläufe zu gewährleisten. Die Form des Leiharbeitseinsatzes als Ad-hoc-Ersatz wird von einer geringen Nutzungsintensität, einem punktuellen, auf wenige Arbeitsbereiche beschränkten Einsatz und einer vergleichsweise kurzen Einsatzdauer charakterisiert. Für die Einsatzbetriebe reduziert der Rückgriff auf Leiharbeit vor allem die Personalsuchkosten. Die Selektion potenzieller Arbeitskräfte wird von den Verleihfirmen übernommen, bei fehlender „Passfähigkeit" oder anderen Schwierigkeiten können die Einsatzbetriebe die überlassenen Arbeitskräfte jederzeit an den Verleiher zurückschicken (Miegel et al. 2007, S. 15; Dudenhöffer/Büttner 2006, S. 30). Auf die arbeitspolitischen Gestaltungsspielräume hat die Nutzung der Leiharbeit als Ad-hoc-Ersatz hingegen kaum Auswirkungen. Aufgrund der geringen Nutzungsintensität und des zeitlich begrenzten Leiharbeitseinsatzes kommt es nur zu punktuellen Interaktionen zwischen Stammkräften und Leiharbeitnehmern. Aus diesem Grund konzentriert sich die anschließende Diskussion der arbeitspolitischen Folgen auf die beiden anderen Einsatztypen. Ein (empirisch jedoch eher seltener) Sonderfall des Adhoc-Ersatzes ist der zeitlich befristete Einsatz von Leiharbeitskräften mit spezifischen Qualifikationen. Anstatt höher qualifizierte Ingenieure, Manager oder Techniker fest einzustellen, greifen Unternehmen auf Leiharbeitskräfte zurück, um sich für einen begrenzten Zeitraum die knappen und damit teuren Qualifikationen und Wissensressourcen der Arbeitskräfte zu sichern.

\subsection{FLEXIBILITÄTSPUFFER}

Die zweite Nutzungsstrategie ist die des Flexibilitätspuffers. Die numerische Flexibilität der Leiharbeit fungiert bei diesem Typus als Anpassungsinstrument an reale Schwankungen des Auftragsvolumens (Kalleberg 2003, S. 157). Leiharbeitskräfte werden in den Betrieb geholt, wenn das Auftragsvolumen aufgrund saisonaler, zyklischer oder anderer Nachfragegründe kurzfristig ansteigt (ähnlich: Promberger et al. 2006, S. 92ff.). Der Leiharbeitseinsatz ist nicht auf Dauer gestellt, sondern bleibt in der Regel auf einen klar eingegrenzten Zeitraum beschränkt. ${ }^{2}$ In den Fallbetrieben schwankt die Einsatzdauer in Abhängigkeit von der Fertigungsstruktur und dem Auftragsvolumen zwischen wenigen Tagen und mehreren Monaten. Folgerichtig ist auch die Nutzungsintensität relativ volatil: $\mathrm{Zu}$ Spitzenzeiten werden in einzelnen Fallbetrieben durchaus Leiharbeitsquoten von $20 \%$ oder gar $30 \%$ erreicht; diese nehmen, sobald das Auftragsvolumen wieder rückläufig ist, jedoch auch schnell wieder ab. Durch den Leiharbeitseinsatz reduzieren die Einsatzbetriebe vor allem die Suchund Einstellungskosten. Die Verleihfirmen übernehmen die Selektion und Rekrutierung potenzieller Arbeitskräfte. Unsere Fallstudien zeigen jedoch, dass sich für die Personalabteilungen der Steuerungs- und Koordinationsaufwand in den Beziehungen zu den Verleihfirmen erhöht: Da der kurzfristige Bedarf an Leiharbeitskräften häufig sehr hoch ist und einzelne Verleih- firmen nicht die Verfügbarkeit über die erforderliche Anzahl an Leiharbeitskräften gewährleisten können, arbeiten die Fallbetriebe in der Regel mit mehreren Verleihfirmen zusammen.

\subsection{STRATEGISCHE NUTZUNG}

Die dritte und historisch jüngste Nutzungsstrategie ist die strategische Nutzung der Leiharbeit (Theodore/Peck 2002, S. 471; Promberger et al. 2006, S. 109ff.). Die Einsatzbetriebe nutzen die numerische Flexibilität der Leiharbeit bei diesem Einsatztypus nicht mehr rein reaktiv als personalpolitisches Anpassungsinstrument an reale Schwankungen des Personalbestandes (Ad-hoc-Ersatz) oder des Auftragsvolumens (Flexibilitätspuffer), sondern strategisch als Instrument der Ungewissheitskontrolle. In Kombination mit einer „Personalpolitik der unteren Linie“ wird Leiharbeit eingesetzt, um die Auswirkungen der zukünftigen und damit ungewissen Entwicklungen der Produktmärkte auf die Profitabilität und damit die Kapitalrendite zu kontrollieren. Zusammen mit anderen Instrumenten der Externalisierung des Beschäftigungsrisikos (Werkverträge, Gewerke, interne Fremdvergabe) stellt Leiharbeit ein Sicherheitsnetz gegen das „Kapazitätsrisiko" der Absatzmärkte dar. Für den Einsatzbetrieb hebelt der Leiharbeitseinsatz faktisch den gesetzlichen Kündigungsschutz aus. Entlassungskosten können vermieden werden, für die Leiharbeitskräfte müssen weder Sozialpläne aufgestellt noch Abfindungen gezahlt werden. Mit diesem Funktionswandel der Leiharbeit von einem reaktiven Anpassungsinstrument hin zu einem Instrument der strategischen Unternehmens- oder Konzernführung ${ }^{3}$ ist zwangsläufig auch ein Formwandel des Leiharbeitseinsatzes verbunden: Die Leih-

\footnotetext{
2 Das schließt nicht aus, dass sich, wie im Fall des untersuchten Großmaschinenbaubetriebs, durch den Eingang weiterer Aufträge die Dauer eines laufenden Einsatzes verlängern kann. Charakteristisch ist für den Typus des Flexibilitätspuffers, dass der Einsatz zu jedem Zeitpunkt zeitlich präzise eingegrenzt und nicht in die Zukunft offen ist.

3 Auf die Aufwertung der Leiharbeit zu einem Instrument der strategischen Unternehmens- oder Konzernführung bezieht sich die Bezeichnung der dritten Nutzungsstrategie als strategische Nutzung. Auch die Nutzung der Leiharbeit als Flexibilitätspuffer oder als Ad-hoc-Ersatz kann durchaus strategisch motiviert sein, Form und Funktion des Leiharbeitseinsatzes werden jedoch vom reaktiven Charakter des Leiharbeitseinsatzes geprägt.
} 
arbeitskräfte werden zu einem mehr oder weniger festen Bestandteil der Belegschaft des Einsatzbetriebs (Kalleberg 2001, S. 488). In den Betriebsfällen des Samples, die Leiharbeit strategisch nutzen, liegt der Leiharbeitsanteil an der Belegschaft zwischen $5 \%$ und $30 \%$. Tendenziell werden die Personalabteilungen der Einsatzbetriebe administrativ entlastet, da aufgrund der relativen Stabilität der Nutzungsintensität nur in Ausnahmefällen Leiharbeitskräfte in größerer Zahl rekrutiert werden müssen. Zudem übernehmen die Disponenten der Verleihfirmen Teile der Steuerung und Kontrolle der Arbeit im Einsatzbetrieb entweder in Form des On-Site-Managements oder durch regelmäßige Besuche im Einsatzbetrieb (Forde 2001, S. 637; Purcell et al. 2004, S. 709f.). In unseren Fallbetrieben wird die Steuerung und Kontrolle der „Atmungsfähigkeit“ der Betriebsorganisation zur zentralen Aufgabe der Personalabteilung: Durch genau dosierte Übernahmen und soziale Integrationsmaßnahmen wird versucht, die Motivation der Leiharbeitskräfte aufrechtzuerhalten und damit die unerwünschte Abwanderung zu verhindern - ohne dabei jedoch die unauflöslich mit der Funktion des Leiharbeitseinsatzes verbundene Statussegmentierung aufzuheben.

\section{1 \\ Fallbeispiel: Leiharbeit als Flexibilitätspuffer}

Der Fallbetrieb, ein Großmaschinenbaubetrieb, ist der Heimatstandort eines inzwischen global agierenden Konzerns. Am untersuchten Standort sind 550 Mitarbeiter beschäftigt, davon 350 in der Fertigung. Zum Zeitpunkt der Untersuchung wurden ungefähr 50 Leiharbeiter im Betrieb eingesetzt, die sich relativ gleichgewichtig auf die Arbeiter- und Angestelltenbereiche verteilten. Die betriebliche Leiharbeitsquote schwankt stark in Abhängigkeit vom Auftragsvolumen. Das Flexibilisierungsinstrument Leiharbeit wird von der Personalabteilung und den direkten Vorgesetzten als reaktives Anpassungsinstrument an die zyklischen Schwankungen der weltweiten Nachfrage nach Großmaschinen eingesetzt. „Von der Kapazität her schaffen wir das (den kurzfristigen Anstieg des Auftragsvolumens, $\mathrm{HH}$ ) gar nicht mit unseren eigenen Kräften“ (PA). Die Arbeitsprozes- se werden von der für einen Leiharbeitseinsatz als Flexibilitätspuffer charakteristischen Tätigkeitssegmentierung geprägt: Damit der Produktionsausstoß schnell ohne größere zeitliche Verzögerung erhöht werden kann, weisen die produktionsverantwortlichen direkten Vorgesetzten den Leiharbeitskräften in ihrem Zuständigkeitsbereich einfache Routinearbeiten zu, die nur geringe Anlernzeiten erfordern, aber die Stammkräfte entlasten und somit die Produktivität erhöhen. Anders als in den Vergleichsbetrieben bildet sich die Tätigkeitssegmentierung zwischen Stammkräften und Leiharbeitern im Großmaschinenbaubetrieb nicht wie im Idealbild der „flexiblen Firma“ (Atkinson 1984) auf der Ebene der Gesamtorganisation heraus, sondern in dezentraler Form auf der Ebene der Einzelabteilungen. Aufgrund des hohen Anteils der kundenbedarfsorientierten Einzel- und Kleinserienfertigung werden auch Ingenieure und Techniker in den Entwicklungs- und Beratungsabteilungen als Leiharbeitskräfte beschäftigt. Unabhängig von ihrem Qualifikationsniveau werden jedoch alle Leiharbeiter in den jeweiligen Abteilungen für einfachere Arbeitsgänge eingesetzt.

Im Ergebnis ist die Belegschaft des Großmaschinenbaubetriebs durch den Leiharbeitseinsatz tief gespalten: Die Stammkräfte, überwiegend im Betrieb ausgebildete Facharbeiter, bilden die Kernbelegschaft, die über ein vergleichsweise hohes $\mathrm{Maß}$ an Beschäftigungssicherheit und über langfristige Aufstiegsperspektiven verfügt. Dagegen zählen die Leiharbeitskräfte zur flexiblen Randbelegschaft. Sie werden unabhängig von ihrem Qualifikationsniveau für stärker standardisierte Routinearbeiten eingesetzt. Auch die Qualifiziertesten unter den Leiharbeitskräften nehmen kaum an den betrieblichen Qualifizierungsmaßnahmen teil. Dies ist zum einen in der Tatsache begründet, dass die Leiharbeiter in aller Regel für Tätigkeiten eingeplant werden, für die verhältnismäßig wenig betriebsspezifisches Wissen erforderlich ist. Zum anderen steht aber auch die spezifische Logik des zeitlich befristeten Leiharbeitseinsatzes als Flexibilitätspuffer einer umfassenden Beteiligung der Leiharbeitskräfte an den betrieblichen Qualifizierungsmaßnahmen entgegen. Um ein Abfließen von betriebsspezifischem Wissen zu verhindern, konzentrieren die direkten Vorgesetzten die Qualifizierungsmaßnahmen auf die Stammkräfte. Und auch in das Ent- geltsystem des Großmaschinenbaubetriebs sind die Leiharbeitskräfte bislang nicht einmal partiell integriert: Sie partizipieren weder an dem betrieblichen Prämiensystem noch erhalten sie Zulagen zu ihren tariflichen Stundenentgelten.

Die Übernahmechancen der Leiharbeitskräfte sind gering. Parallel zum Leiharbeitseinsatz rekrutiert die Personalabteilung Arbeitskräfte direkt vom externen Arbeitsmarkt. Obwohl sie sich ihrer geringen Chancen bewusst sind, hoffen alle von uns interviewten Leiharbeitskräfte auf die Übernahme in ein festes Beschäftigungsverhältnis. Die Mehrheit hat eine Vielzahl erfolgloser Bewerbungen hinter sich und der direkte Weg zurück in den ersten Arbeitsmarkt scheint blockiert: „Beim Arbeitsamt bekommst du nur noch Adressen von Zeitarbeitsfirmen" (LA). Ihnen ist bewusst, dass sie ,jederzeit auf die Straße geworfen werden" (LA) können. Die hohe Unsicherheit eines Leiharbeitsverhältnisses macht sie verwundbar und wirkt vor dem Hintergrund des für sie blockierten Arbeitsmarkts disziplinierend und individualisierend. Um ihre geringen Übernahmechancen nicht zu gefährden, fügen sie sich weitgehend widerspruchslos in die Betriebsgemeinschaft ein und versuchen die direkten Vorgesetzten durch permanente, „freiwillige“ Leistungsverausgabung zu beeindrucken:

„Ich streng mich schon an, meinen Job gut zu machen. Ich hab mich vom ersten Tag bemüht. Mein Chef sagt: Das ist eine Chance, du musst Dich hier jeden Tag aufs Neue bewerben" (LA).

Dabei nehmen die Leiharbeitskräfte auch in Kauf, dass sie als Mitglieder der Randbelegschaft einfachere Tätigkeiten verrichten und von den Kollegen und Vorgesetzten nur wenig Anerkennung für ihre Arbeit erfahren.

Auf die Stammbelegschaft des Großmaschinenbaubetriebs hat der Leiharbeitseinsatz hingegen bislang nur geringe Auswirkungen. Die Mehrheit der Stammkräfte sieht - auch aufgrund der überaus positiven wirtschaftlichen Entwicklung des Betriebs zum Zeitpunkt der Untersuchung - in den Leiharbeitskräften keine Konkurrenten auf Augenhöhe, obwohl sie formal häufig über die gleichen Qualifikationen verfügen. „Ich denke, die meisten Zeitarbeiter haben keinen festen Job, weil sie nicht qualifiziert sind. Das ist ein ganz großes Manko von ihnen, eigentlich das größte Manko“ (SK). Welche Folgen die 
auch bei den Stammkräften durchaus vorhandenen latenten Prekarisierungsängste in Zeiten konjunkturellen Niedergangs für die Beziehungen zwischen den Belegschaftsgruppen haben werden, muss an dieser Stelle offen bleiben. Bislang blockieren allerdings die auch für die Leiharbeiter spürbaren Distinktionsstrategien der Stammkräfte zusammen mit der zeitlichen Befristung des Leiharbeitseinsatzes die Solidarisierungsbemühungen, die vom Betriebsrat initiiert werden. Im Falle rückläufigen Auftragsvolumens werden zuerst die Leiharbeitskräfte freigesetzt, bevor die Stundenkonten der Stammkräfte abgeschmolzen werden. Die strikte Tätigkeitssegmentierung zwischen beiden Belegschaftsgruppen führt dazu, dass die kurzfristige Substituierbarkeit der Kernbelegschaft gering ist. Den Leiharbeitskräften fehlt es an betriebsspezifischem Wissen, um die Positionen der Stammkräfte zu übernehmen. Anders stellt sich die Situation für die kleine Gruppe von Stammkräften dar, die in der Logistik und in der Fertigung die gleichen Arbeiten verrichten wie die Leiharbeitskräfte. Ihre Substituierbarkeit ist hoch, da die Leiharbeitskräfte direkt und ohne zusätzliche Anlernzeit ihre Positionen übernehmen können. In diesen Bereichen sind Spannungen zwischen Stammund Leiharbeitskräften an der Tagesordnung.

\section{5 \\ Fallbeispiel: Strategische Nutzung der Leiharbeit}

Der Fallbetrieb, ein Elektromaschinenbaubetrieb, ist Teil eines internationalen Maschinenbaukonzerns. Am untersuchten Standort sind 650 Mitarbeiter beschäftigt, davon 120 als Leiharbeiter. Die Leiharbeitsquote ist seit einigen Jahren relativ stabil, per Betriebsvereinbarung ist eine Obergrenze festgelegt worden. Das Flexibilisierungsinstrument Leiharbeit wird von der Personalabteilung eingesetzt, um ein „Sicherheitsnetz für das Unternehmen " $\mathrm{zu}$ etablieren: „Wenn es mal nicht so gut läuft, können wir eben darüber ganz gut atmen" (PA). Der Leiharbeitseinsatz wird in weiten Teilen der Fertigung von der für die strategische Nutzung der Leiharbeit charakteristischen Aufhebung der Tätigkeitssegmentierung zwischen Stamm- und Leiharbeitskräften geprägt. Um eine, wie durch die
Verwendung der Metapher der „Atmungsfähigkeit" suggeriert, schnelle Anpassung des Produktionsausstoßes sicherzustellen, werden die Leiharbeitskräfte zumindest in der seit einigen Jahren auf Fließfertigung umgestellten Produktion dauerhaft und umfassend in die Arbeitsprozesse integriert. Sie verrichten exakt die gleichen Arbeiten wie die Stammkräfte. Zwar bleibt die Statussegmentierung zwischen Stammund Leiharbeitskräften bestehen, aber aufgrund der Verflechtung im Arbeitsprozess kann von einer Spaltung der Belegschaft in eine Kern- und eine Randbelegschaft keine Rede mehr sein. Die Leiharbeitskräfte bilden eine „flexible Quasi-Stammbelegschaft" (Seifert/Brehmer 2008), die zumindest im Arbeitsprozess der Stammbelegschaft gleichgestellt ist. Damit die Leiharbeitskräfte an den gleichen Arbeitsplätzen eingesetzt werden können wie die Stammkräfte, nehmen sie in vollem Umfang an den betrieblichen Qualifizierungsmaßnahmen teil. Zudem sind die Leiharbeitskräfte partiell in das Entgeltsystem des Einsatzbetriebs eingebunden: Der Betriebsrat hat mit Unterstützung der direkten Vorgesetzten durchgesetzt, dass die Leiharbeitskräfte eine betriebliche Zulage zu ihrem tariflichen Entgelt erhalten und vollständig in das Prämiensystem des Elektromaschinenbaubetriebs integriert sind.

Die Übernahmechancen der Leiharbeiter sind gering, wenn auch etwas höher als im Großmaschinenbaubetrieb. Im Bereich der Fertigung sind die von den Leiharbeitern bekleideten Positionen vollständig in die betrieblichen Aufstiegswege einbezogen, vom externen Arbeitsmarkt werden keine Arbeitskräfte eingestellt. Scheidet eine Stammkraft aus, wird ein bereits eingearbeiteter Leiharbeiter übernommen. Allerdings bleiben Übernahmen selten, die Personalpolitik des Elektromaschinenbaubetriebs ist auf eine Stabilisierung des Verhältnisses zwischen beiden Belegschaftsgruppen ausgerichtet. Trotz der insgesamt geringen Aussichten hoffen alle von uns interviewten Leiharbeitskräfte auf einen festen Arbeitsvertrag im Einsatzbetrieb. Angesichts ihrer geringen Arbeitsmarktchancen hätte die Übernahme für sie den Charakter eines „Fünfers im Lotto“ (LA). Um durch das enge Nadelöhr einer Übernahme zu schlüpfen, versuchen sie, die direkten Vorgesetzten permanent von ihrer Leistungsfähigkeit und Leistungsbereitschaft $\mathrm{zu}$ überzeugen. In der Betriebsleitung gelten die Leiharbeiter, die bereits längere Zeit im Betrieb sind, als „Leistungsträger“ (PA). Allerdings bildet sich unter den Leiharbeitskräften allzu leicht eine Konkurrenz um die knappen Übernahmechancen heraus, die disziplinierend und vor allem individualisierend wirkt. Zwar fühlen sich vor allem die Leiharbeitskräfte, die bereits über Erfahrungen aus anderen Einsatzbetrieben verfügen, verhältnismäßig gut in die soziale Gemeinschaft des Betriebs integriert. Trotzdem nehmen sie sich im Vergleich mit den Stammkräften als „Arbeiter zweiter Klasse" wahr. Täglich spüren sie die Verwundbarkeit, die mit einem Leiharbeitsverhältnis einhergeht, am eigenen Leib:

„Man muss es ja ganz krass sagen: Wenn ein Leiharbeiter nicht funktioniert oder wenn die Qualität nicht ist, dann wird er abbestellt, dann ist er weg, da wird nicht lange rumgefackelt" (LA).

Auch die Befunde aus den anderen Fallbetrieben des Samples deuten darauf hin, dass im Vergleich der Nutzungsstrategien die strategische Nutzung für die Leiharbeitskräfte mit geringfügig gesteigerten Übernahme-, Anerkennungs- und Identifikationschancen verbunden ist. Noch gröBer sind jedoch die Unterschiede zwischen der Leiharbeitsnutzung als Flexibilitätspuffer und der strategischen Nutzung in Bezug auf die potenziellen Rückwirkungen auf die weiterhin formal sicher beschäftigten Stammkräfte. Die Aufhebung der Tätigkeitssegmentierung weitet im Elektromaschinenbaubetrieb wie in den anderen Fallbetrieben des Samples die strukturellen Voraussetzungen für eine konkurrenzorientierte Steuerung der Arbeit erheblich aus. Denn dort, wo Stammkräfte und Leiharbeiter über die gleichen Qualifikationen verfügen und die gleichen Tätigkeiten verrichten, ist die kurzfristige Substituierbarkeit der Stammkräfte hoch - relativ unabhängig von ihrer Betriebszugehörigkeit. Selbst die Positionen der qualifizierten Stammkräfte mit betriebsspezifischem Wissen können direkt von den Leiharbeitskräften übernommen werden. Die Stammkräfte sind sich ihrer Ersetzbarkeit durchaus bewusst, schließlich „macht man dasselbe und hat eigentlich denselben Werdegang " (SK). Auf diese Weise trägt der Leiharbeitseinsatz zu einer Entwertung des betrieblichen Erfahrungswissens der langjährigen Stammkräfte bei:

„Die Frage ist, wenn es nicht mehr so boomt, dann bin ich eigentlich sehr skeptisch bei der Sache... Es kann natürlich kommen, dass die sagen: ,Du musst gehen, 
weil der andere billiger ist. ' Da habe ich halt Pech gehabt, ganz großes Pech. Das weiß ich nicht, inwieweit das beim Arbeitgeber noch einen Wert hat, wenn man mit der ersten Stunde dabei war, ob das noch was zählt in der heutigen Zeit oder ob man da drüber hinweggeht (SK).“

Das nicht nur diffuse, sondern sehr konkrete Gefühl der Ersetzbarkeit wirkt disziplinierend auf die Stammkräfte. Während in der Vergangenheit Aufstiegs- und Qualifizierungsplätze entsprechend dem Status der Beschäftigten verteilt wurden, herrscht heute eine statusunabhängige Konkurrenz um knappe Qualifizierungsund Aufstiegsperspektiven. Verstärkt wird die disziplinierende Wirkung der Konkurrenz durch die Erinnerung an die letzten „Entlassungswellen“ Anfang des neuen Jahrtausends - eine Erfahrung, die aktuell wieder sehr präsent sein dürfte. Vor diesem Hintergrund wird die Flexibilität der Leiharbeiter mit gemischten Gefühlen wahrgenommen: „Ein Leiharbeiter kommt auf mehr Stationen in drei Jahren, wie ich in 30 Jahren" (SK). Allenfalls die Stammkräfte, die bereits einen kleinen innerbetrieblichen Aufstieg absolviert haben, können sich einer direkten Konkurrenz mit den Leiharbeitskräften und damit auch der disziplinierenden Wirkung des Leiharbeitseinsatzes ein Stück weit entziehen.

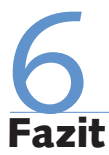

Im Vergleich mit den klassischen Nutzungsstrategien Ad-hoc-Ersatz und Flexibilitätspuffer wandelt sich die betriebliche Funktion des Flexibilisierungsinstruments Leiharbeit bei der strategischen Nutzung von einem reaktiven personalpolitischen Anpassungsinstrument hin zu einem Instrument der strategischen Unternehmensführung. Mit Hilfe eines umfassenden und auf Dauer gestellten Leiharbeitseinsatzes wird ein Sicherheitsnetz gegen das „Kapazitätsrisiko" der Absatzmärkte errichtet. Die veränderte Funktion des Flexibilisierungsinstruments Leiharbeit geht zwangsläufig mit einem Wandel der Form des Leiharbeitseinsatzes einher. Werden den Leiharbeitskräften im Falle zeitlich befristeter Einsätze überwiegend randständige Tätigkeiten zugewiesen, die nur eine kurze Anlernzeit erfordern, sind beide Belegschaftsgruppen bei der strategischen Nut- zung der Leiharbeit im Arbeitsprozess weitgehend verflochten. In allen Fallbetrieben unseres Samples, die Leiharbeit strategisch nutzen, verrichten Stamm- und Leiharbeitskräfte in großen Bereichen der Betriebsorganisation die gleichen Arbeiten. Die Statussegmentierung bleibt jedoch bestehen, schließlich basiert die Funktion der Leiharbeit als „Sicherheitsnetz“ für die Kapitalrendite gerade auf der Aushebelung des gesetzlichen Kündigungsschutzes. Dagegen geht bei der Nutzungsstrategie als Flexibilitätspuffer die Statussegmentierung mit einer mehr oder weniger strikten Tätigkeitssegmentierung einher: Nur wenige Stammkräfte verrichten in den Randbereichen des Betriebs die gleichen Tätigkeiten wie die nur zeitlich befristet eingesetzten Leiharbeitskräfte.

Die Gleichstellung der Stammkräfte und der Leiharbeiter im Arbeitsprozess durch die strategische Nutzung der Leiharbeit hat weitreichende arbeitspolitische Folgen. Gewissermaßen im Windschatten der veränderten Funktion von Leiharbeit wird im Einsatzbetrieb strukturell ein Mechanismus verankert, der - vermittelt über die Leiharbeitskräfte - auch die langjährigen Beschäftigten stärker an die allgemeine Arbeitsmarktentwicklung koppelt. Während bei einem Einsatz als Flexibilitätspuffer die Belegschaft des Einsatzbetriebs tief in eine Kern- und eine Randbelegschaft gespalten ist, hat bei der strategischen Nutzung jede Stammkraft im Extremfall einen Leiharbeiter vor Augen, der die gleichen Tätigkeiten verrichtet und für den die Übernahme in ein festes Arbeitsverhältnis im Einsatzbetrieb ein erstrebenswertes Ziel ist. Die betriebliche Rekrutierungspraxis verdeutlicht, dass durch die strategische Nutzung der Leiharbeit die kurzfristige Substituierbarkeit der Stammkräfte erhöht wird. Als Ersatz für abgewanderte Stammkräfte werden bereits eingearbeitete Leiharbeiter übernommen, deren Leistungsfähigkeit und Leistungsbereitschaft die direkten Vorgesetzten überzeugt hat. Direkt vom externen Arbeitsmarkt werden zumindest in den Fertigungsbereichen in keinem unserer Fallbetriebe Arbeitskräfte rekrutiert, sodass der Weg in den Einsatzbetrieb nur noch über ein Leiharbeitsverhältnis führt. Dagegen werden in allen Fallbetrieben des Samples, in denen Leiharbeit als Flexibilitätspuffer eingesetzt wird, parallel zum Leiharbeitseinsatz Arbeitskräfte vom externen Arbeitsmarkt rekrutiert.
Die Ergebnisse unserer Fallstudien zeigen, dass durch die strategische Nutzung die Reichweite und die Form der Disziplinierungseffekte der Leiharbeit erheblich ausgeweitet werden. Unabhängig von der betrieblichen Nutzungsstrategie macht die Unsicherheit ihres Beschäftigungsverhältnisses die Leiharbeiter verwundbar. Für die meisten von ihnen stellt die Übernahme in ein festes Arbeitsverhältnis im Einsatzbetrieb die einzige realistische Chance auf eine dauerhafte Rückkehr in den ersten Arbeitsmarkt dar. Als Leiharbeiter werden sie zwar in den Arbeitsprozessen des Einsatzbetriebes eingesetzt, mit einem Bein stehen sie jedoch permanent in der Erwerbslosigkeit. Werden sie vom Einsatzbetrieb an die Verleihfirma zurückgeschickt und hat diese keine alternativen Einsatzmöglichkeiten, dann droht ihnen die betriebsbedingte Kündigung. Diese latente Drohung im Hinterkopf, setzen sie nahezu alles daran, im Einsatzbetrieb in ein reguläres Arbeitsverhältnis übernommen zu werden, auch wenn es zunächst nur ein befristetes ist. Allerdings bleiben die Disziplinierungseffekte nicht allein auf die Leiharbeitskräfte begrenzt. Im Vergleich mit dem Typus des Flexibilitätspuffers ist die strategische Nutzung mit einer erheblichen Ausweitung der möglichen Disziplinierungseffekte verbunden. Durch die Gleichstellung beider Belegschaftsgruppen in den betrieblichen Arbeitsprozessen beschleicht die Stammkräfte nicht mehr nur ein diffuses Gefühl der Ersetzbarkeit, sondern die Austauschbarkeit ist sehr konkret erfahrbar. In den von uns untersuchten Fallbetrieben waren sich alle interviewten Stammkräfte ihrer kurzfristigen Substituierbarkeit durch Leiharbeiter bewusst - unabhängig von der Dauer ihrer Betriebszugehörigkeit. Während, wie von der frühen Prekarisierungsforschung herausgearbeitet, bei einem Leiharbeitseinsatz als Flexibilitätspuffer die Leiharbeiter vor allem die latente Drohung des sozialen Abstiegs und Statusverlusts symbolisieren, können bei der strategischen Nutzung beide Belegschaftsgruppen in ein direktes Konkurrenzverhältnis um Aufstiegs-, Qualifizierungs- und Beschäftigungschancen gesetzt werden.

Einschränkend muss jedoch darauf hingewiesen werden, dass die strategische Nutzung zwar mit einer deutlichen Erhöhung des Disziplinierungsrisikos für die Stammkräfte einhergeht, dass aber von einem Disziplinierungsautomatismus auch keine Rede sein kann. Unsere Projektergeb- 
nisse deuten auch darauf hin, dass die Schnittstelle zwischen Stammkräften und Leiharbeitern betriebspolitisch sehr wohl gestaltbar ist. Wie sich die Gleichstellung beider Belegschaftsgruppen in den Arbeitsprozessen im Betrieb auswirkt, ist vom Verhalten der betrieblichen Akteure sowie von der allgemeinen wirtschaftlichen Lage und der Marktposition des Betriebs abhängig. Darüber hinaus bringt die enge Verflechtung von Stammkräften und Leiharbeitern im Arbeitsprozess auch neue Solidarisierungspotenziale mit sich, die von den betrieblichen Interessenvertretern mobilisiert werden können, um diese Schnittstelle betriebspolitisch zu gestalten. In den Betrieben, in denen Betriebsräte die Stammbe- legschaften für die Probleme und Anliegen der Leiharbeitskräfte sensibilisieren und deren Interessen in ihre Interessenvertretungspraxis integrieren, besteht die Möglichkeit einer statusgruppenübergreifenden Solidarisierung, die zumindest temporär Konkurrenzen überwindet. An diesem Punkt besteht jedoch weiterer Forschungsbedarf. Anliegen dieses Beitrags war es, deutlich zu machen, dass mit dem Funktionswandel der Leiharbeit - in der Regel als Nebenfolge, in Einzelfällen auch intendiert - strukturell ein Mechanismus etabliert wird, der die prekäre Position der Leiharbeitskräfte instrumentalisiert, um die betrieblichen Arbeitsmärkte der Einsatzbetriebe ein Stück weit von innen zu öffnen, ohne dass ihre externe Geschlossenheit verloren geht. Die Arbeit auch der Stammkräfte mit langer Betriebszugehörigkeit wird stärker an die allgemeinen Arbeitsmarktentwicklungen gekoppelt. Betriebspolitisch mag sich dieser strukturell mit der strategischen Nutzung der Leiharbeit verbundene Mechanismus zwar temporär stillstellen lassen, dauerhaft auflösen lässt er sich allein auf der betrieblichen Ebene jedoch nicht. Dazu wäre nur eine konsequente Politik der Entprekarisierung in der Lage, die durch eine Reform der rechtlichen Rahmenbedingungen des Leiharbeitseinsatzes und der sozialen Sicherungssysteme die Positionen der Leiharbeitskräfte im Arbeitsmarkt stabilisiert.

\section{LITERATUR}

Atkinson, J. (1984): Manpower strategies for flexible organisations, in: Personel Management 8, S. 28-31

Beaud, S./Pialoux, M. (2004): Die verlorene Zukunft der Arbeiter, Konstanz

Bellmann, L./Kühl, A. (2007): Weitere Expansion der Leiharbeit? Eine Bestandsaufnahme auf Basis des IAB-Betriebspanels, Düsseldorf Brinkmann, U./Dörre, K./Röbenack, S. (2006): Prekäre Arbeit. Ursachen, Ausmaß, soziale Folgen und subjektive Verarbeitungsformen unsicherer Beschäftigungsverhältnisse, Bonn

Broschak, J. P./Davis-Blake, A. (2006): Mixing Standard Work and Nonstandard Deals: the Consequences of Heterogeneity in Employment Arrangements, in: Academy of Management Journal 2, S. 371-393

Carnoy, M./Castells, M./Benner, C. (1997): Labour markets and employment practices in the age of flexibility: A case study of Silicon Valley, in: International labour review 1, S. 47-48

Castel, R,/Dörre, K. (2009): Prekarität, Abstieg, Ausgrenzung. Die soziale Frage am Beginn des 21. Jahrhunderts, Frankfurt a. $M$.

Dörre, K./Holst, H. (2007): Funktionswandel von Zeitarbeit, Projektantrag Otto-Brenner-Stiftung, Frankfurt a. M.

Dörre, K./Kraemer, K./Speidel, F. (2004): Prekäre Arbeit, in: Das Argument 245, S. 378-397

Dudenhöffer, F./Büttner, C. (2006): Der Wettbewerbsfaktor Zeitarbeit in der Automobilindustrie, in: Ifo Schnelldienst 59, S. 30-36

Forde, C. (2001): Temporary Arrangements: the Activities of Employment Agencies in the UK, in: Work, Employment and Society 3, S. 631-644
Kalleberg, A. L. (2003): Flexible Firms and Labor Market Segmentation. Effects of Workplace Restructuring on Jobs and Workers, in: Work and Occupations 2, S. 154-175

Kraemer, K./Speidel, F. (2004): Prekäre Leiharbeit. Zur Integrationsproblematik einer atypischen Beschäftigungsform, in: Vogel, B. (Hrsg.) (2004): Leiharbeit: Neue sozialwissenschaftliche Befunde zu einer prekären Beschäftigungsform, Hamburg, S. 119-153

Miegel, M./Wagl, S./Schulte, M. (2007): Die Rolle der Zeitarbeit in einem sich ändernden Arbeitsmarkt, Bonn

Promberger, M. (2006): Leiharbeit - Flexibilität und Prekarität in der betrieblichen Praxis, in: WSI-Mitteilungen 5, S. 263-269

Promberger, M./Bellmann, L./Dreher, C./Sowa, F./Schramm, S./Theuer, S. (2006); Leiharbeit im Betrieb - Strukturen, Kontexte und Handhabung einer atypischen Beschäftigungsform, Nürnberg

Purcell, J. (2004): Temporary Work Agencies: Here Today, Gone Tomorrow?, in: British Journal of Industrial Relations 4, S. 705-725

Seifert, H./Brehmer, W. (2008): Leiharbeit: Funktionswandel einer flexiblen Beschäftigungsform, in: WSI Mitteilungen 6, S. 335-341 Theodore, N./Peck, J. (2002): The Temporary Staffing Industry: Growth Imperatives and Limits to Contingency, in: Economic Geography 4, S. 463-493

Vogel, B. (2004): Leiharbeit: Neue sozialwissenschaftliche Befunde zu einer prekären Beschäftigungsform, Hamburg Wassermann, W./Rudolph, W. (2007): Leiharbeit als Gegenstand betrieblicher Mitbestimmung, Düsseldorf 